\title{
Plantas alimentícias silvestres aquáticas: prospecção científica sobre o potencial nutricional negligenciado
}

\author{
Aquatic forest food plants: scientific prospection on neglected nutritional potencial
}

Plantas acuáticas de alimentos forestales: prospección científica del potencial nutricional descubierto

\author{
Mirna Andrade Bezerra \\ ORCID: https://orcid.org/0000-0003-2617-5477 \\ Universidade Federal do Piauí, Brasil \\ E-mail: mirna.a.bez11@gmail.com \\ Francisco Soares Santos Filho \\ ORCID: https://orcid.org/0000-0002-1713-7228 \\ Universidade Estadual do Piauí, Brasil \\ E-mail: fsoaresfilho@gmail.com
}

\begin{abstract}
Resumo
As plantas alimentícias silvestres oferecem possibilidades de diversificação nutricional e econômica. São consideradas como espécies daninhas e crescem espontaneamente em jardins, terrenos baldios, entre outros ambientes inclusive os aquáticos. As plantas aquáticas também podem ser inseridas como recurso alimentício com rico potencial.Foi realizada prospecção científica durante o mês de Outubro de 2020, com base na busca de artigos científicos que tratem sobre a utilização de plantas alimentícias aquáticas e a sua composição nutricional nas bases de dados Web of Science, Science direct e Pubmed.Adicionalmente, foram realizadas buscas no livro Plantas Alimentícias Não Convencionais (PANC) sobre plantas alimentícias aquáticas com ocorrência e utilização no Brasil.Um total de 81 artigos foi computado para as palavras-chave e respectivas combinações inseridas no campo de busca. Após a análise mais aprofundada de artigos de interesse nenhum deles apresentou conteúdo sobre plantas alimentícias silvestres e aquáticas e a sua composição nutricional. No entanto, foram identificadas pesquisas que tratam sobre plantas terrestres e aquáticas utilizadas na alimentação e a relação estabelecida entre homem-ambiente em condições de vulnerabilidade. A ampliação da identificação e uso de espécies alimentícias, bem com os valores nutricionais agregados às pesquisas permitem a inserção das plantas em dietas sustentáveis. A escassez de estudos nesta área permite a visualização de lacunas no conhecimento e amplas possibilidades de pesquisa promissoras no conhecimento sobre a biodiversidade.
\end{abstract}

Palavras-chave: Plantas alimentícias não convencionais; Segurança alimentar; Diversificação alimentar; Composição nutricional.

\begin{abstract}
Wild food plants offer possibilities for nutritional and economic diversification. They are considered as harmful species and grow spontaneously in gardens, vacant lots, among other environments including aquatic ones. Aquatic plants can also be inserted as a food resource with rich potential. Scientific research was carried out during the month of October 2020, based on the search for scientific articles dealing with the use of aquatic food plants and their nutritional composition in the Web of Science, Science direct and Pubmed databases. Additionally, searches were carried out in the book Non-Conventional Food Plants (PANC) on aquatic food plants occurring and used in Brazil. A total of 81 articles were computed for the keywords and respective combinations entered in the search field. After a more in-depth analysis of articles of interest, none of them presented content on wild and aquatic food plants and their nutritional composition. However, research has been identified that deals with terrestrial and aquatic plants used in food and the relationship established between man and the environment in vulnerable conditions. The expansion of the identification and use of food species, as well as the nutritional values added to research, allow the insertion of plants in sustainable diets. The scarcity of studies in this area allows the visualization of gaps in knowledge and ample possibilities for promising research in knowledge about biodiversity.
\end{abstract}

Keywords: Unconventional food plants; Food security; Food diversification; Nutritional composition.

\section{Resumen}

Las plantas alimenticias silvestres ofrecen posibilidades de diversificación nutricional y económica. Son consideradas especies nocivas y crecen espontáneamente en jardines, terrenos baldíos, entre otros ambientes, incluidos los acuáticos. Las plantas acuáticas también pueden insertarse como un recurso alimenticio con un gran potencial. La 
prospección científica se realizó durante octubre de 2020, a partir de la búsqueda de artículos científicos que aborden el uso de plantas acuáticas alimentarias y su composición nutricional en las bases de datos. Web of Science, Science direct y datos de Pubmed. Adicionalmente, se realizaron búsquedas en el libro Non-Conventional Food Plants (PANC) sobre plantas acuáticas alimentarias presentes y utilizadas en Brasil. Se computaron un total de 81 artículos para las palabras clave y sus combinaciones. el campo de búsqueda. Después de un análisis más profundo de los artículos de interés, ninguno de ellos presentó contenido sobre plantas alimenticias silvestres y acuáticas y su composición nutricional. Sin embargo, se han identificado investigaciones que tratan sobre plantas terrestres y acuáticas utilizadas en la alimentación y la relación que se establece entre el hombre y el medio ambiente en condiciones de vulnerabilidad. La expansión de la identificación y uso de especies alimentarias, así como los valores nutricionales agregados a la investigación, permiten la inserción de plantas en dietas sostenibles. La escasez de estudios en esta área permite visualizar lagunas en el conocimiento y amplias posibilidades para investigaciones prometedoras en el conocimiento sobre la biodiversidad.

Palabras clave: Plantas de alimentos no convencionales; Seguridad alimentaria; Diversificación alimentaria; Composición nutricional.

\section{Introdução}

As relações estabelecidas entre os seres humanos e as plantas permitiram o desenvolvimento e diversificação de ambos, quanto aos usos e modos de preparo para inserção na alimentação cotidiana. Com o passar do tempo, com o advento da agricultura, a ampla diversidade de vegetais disponíveis e utilizados, foi sendo restringida a culturas monoespecíficas. (Raven, et al., 2014; Hora, et al., 2020)

Vários fatores podem explicar a seleção de determinadas espécies alimentícias para o cultivo em larga em escala, como por exemplo, a disponibilidade do recurso, o sabor, a facilidade de colheita, condições sociais, dentre outros aspectos. No entanto, “muitas plantas consideradas "daninhas", "inços", "pragas”, “invasoras”, "espontâneas”, "ruderais", encontradas em jardins, permeando espécies cultivadas ou em terrenos baldios, são espécies com grande valor ecológico (Kinupp \& Barros, 2004), algumas são alimentícias, oferecem possibilidades de diversificação nutricional e econômica.

O desenvolvimento de estudos científicos sobre plantas alimentícias silvestres ainda é baixo. Porém, no Brasil há pesquisas importantes sobre a temática, em ambientes onde a escassez de recursos e períodos de secas prolongados podem ter subsidiado estudos na região semi-árida. Nascimento et al. (2012), Nascimento et al. (2013), Cruz et al. (2014), Chaves et al. (2015), Chaves et al. (2017) e Gomes et al. (2020), dentre outros pesquisadores retratam a biodiversidade alimentícia disponível e negligenciada em ambientes tradicionalmente reconhecidos como pobres, a exemplo do Nordeste brasileiro.

Vale ressaltar, que houve um interesse maior por essa temática a partir de 2008 (Jacob, et al., 2020) e a utilização do termo Plantas Alimentícias Não Convencionais (PANC), por Valdelly Kinnup, proporcionou a popularização sobre os usos e possibilidades não somente de plantas alimentícias silvestres, como também espécies vegetais de uso convencional, mas que possuíam outras partes subutilizadas, com igual ou superior valor nutricional comparados com os órgãos vegetativos tradicionalmente consumidos. O termo PANC é bastante difundido no Brasil, no entanto, internacionalmente é mais comum utilizar plantas alimentícias silvestres para designar as espécies "não cultivadas que podem ser coletadas em ambientes naturais" (Hora, et al., 2020).

Destaca-se ainda, inserida no contexto do baixo conhecimento sobre a biodiversidade disponível, as plantas aquáticas que podem ser inseridas como recurso alimentício com rico potencial. Kinupp e Barros (2008) ressaltam em seus estudos, que as plantas aquáticas são fontes promissoras de proteína e destacam pelo menos três espécies encontradas na região metropolitana de Porto Alegre com possibilidades de uso como verduras ou no preparo de concentrados protéicos.

A provável condição alimentícia das plantas aquáticas também foi evidenciada em outros locais do mundo, como por exemplo, no nordeste da Tailândia. Cruz-Garcia et al. (2016), apontaram espécies utilizadas na alimentação, que crescem espontaneamente entre os cultivares de arroz, sendo apontado como recurso importante para populações em ocasiões de escassez de alimentos. 
A problemática não está conferida somente ao desconhecimento sobre as plantas de uso alimentício terrestres e aquáticas. Jacob e Albuquerque (2020) ressaltam que há quatro lacunas a serem preenchidas e que podem apontar caminhos para inserção destas plantas em dietas sustentáveis, com a finalidade de conhecer a biodiversidade de plantas e componentes nutricionais que proporcionem benefícios a saúde da população. Uma dessas lacunas está relacionada a falta de informações nutricionais, modos de preparo, composição química e componentes tóxicos nas pesquisas realizadas até então sobre plantas alimentícias silvestres e/ou PANC.

A indisponibilidade destes dados funciona como uma barreira na popularização das plantas, restringindo o uso a pequenas populações locais. Tendo em vista a relevância desta temática, objetivou-se prospectar trabalhos científicos nacionais e internacionais sobre a composição nutricional de plantas alimentícias silvestres e/ou PANC aquáticas para utilização em dietas humanas, bem como apontar espécies aquáticas com potencial alimentício ou uso confirmado no Brasil.

\section{Metodologia}

A pesquisa realizada é de natureza quantitativa, pois há a coleta de dados quantitativos ou numéricos (Pereira, et al., 2018). Para coleta de dados a prospecção científica foi realizada durante o mês de Outubro de 2020, com base na busca de artigos científicos que tratem sobre a utilização de plantas alimentícias aquáticas e a sua composição nutricional. Adicionalmente, foram realizadas buscas no livro Plantas Alimentícias Não Convencionais (Kinupp \& Lorenzzi, 2014) sobre plantas alimentícias aquáticas com ocorrência e utilização no Brasil.

As palavras-chave utilizadas foram: Food plants (Plantas alimentícias), wild (Selvagem/silvestre), Aquatics (Aquáticas) e Composition nutricional (Composição nutricional). A fim de tornar a busca mais específica, concatenações foram realizadas entre os termos com a inserção do conector "AND". As bases de dados Web of Science, Science direct e Pubmed foram selecionadas por agregarem maior amplitude de trabalhos científicos.

Foram selecionados apenas os artigos de pesquisa publicados nos últimos 20 anos que apresentassem os descritores em seus títulos e/ou resumos. A justificativa para a delimitação temporal empregada teve como finalidade revelar como a pesquisa nesta área foi sendo desenvolvida, bem como, a avaliação sobre as perspectivas de crescimento ou redução de estudos na área.

Os critérios de exclusão desta prospecção científica são: 1- Os artigos duplicados encontrados na mesma base de dados e/ou em mais de uma; 2 - as pesquisas que não estão relacionadas aos teores e composição nutricional de plantas alimentícias aquáticas com uso não convencional ou silvestre e 3 - Artigos científicos não relacionados a utilização das espécies alimentícias para seres humanos.

Para a análise dos dados foi utilizado o programa Microsoft Excel versão Windows 10.

\section{Resultados}

Quanto às concatenações "Food plants" and "wild" and "aquatic", a busca inicial com estes descritores na Web of Science resultaram em 4 artigos científicos, 176 na Science direct e 144 na Pubmed. Com relação à segunda combinação de palavras-chave "Food plants" and "wild" and "nutritional composition", foram encontrados 6 registros na Web of Science, 33 no Science direct e 91 no Pubmed. A última busca realizada teve como objetivo encontrar pesquisas que tratassem de plantas alimentícias silvestres, aquáticas e análise da composição nutricional. Desta forma, não foram encontrados estudos científicos com as palavras chave selecionadas na Web of Science, diferentemente da Science direct com 68 registros e Pubmed com 13, como mostra a Tabela 1. 
Tabela 1: Número de artigos científicos publicados nas bases de dados Web of Science, Science Direct e PubMed.

\begin{tabular}{cccc}
\hline Descritores & Web of Science & Science direct & Pubmed \\
\hline $\begin{array}{c}\text { "Food plants" and "wild" and } \\
\text { "aquatic" }\end{array}$ & 4 & 176 & 144 \\
\hline $\begin{array}{c}\text { "Food plants" and "wild" and } \\
\text { "nutritional composition" }\end{array}$ & 6 & 33 & 91 \\
\hline $\begin{array}{c}\text { "food plants" and "wild" and } \\
\text { "aquatic" and "nutritional } \\
\text { composition" }\end{array}$ & 0 & 68 & 13 \\
\hline
\end{tabular}

Fonte: Web of Science, Science direct e Pubmed (2020).

Com a utilização das bases científicas já mencionadas, um total de 81 artigos foram computados para as palavraschave e respectivas combinações inseridas no campo de busca. As estratégias de busca e seleção das pesquisas seguiram as etapas descritas no fluxograma da Figura 1.

Figura 1: Fluxograma com as etapas de análise e seleção de artigos científicos.

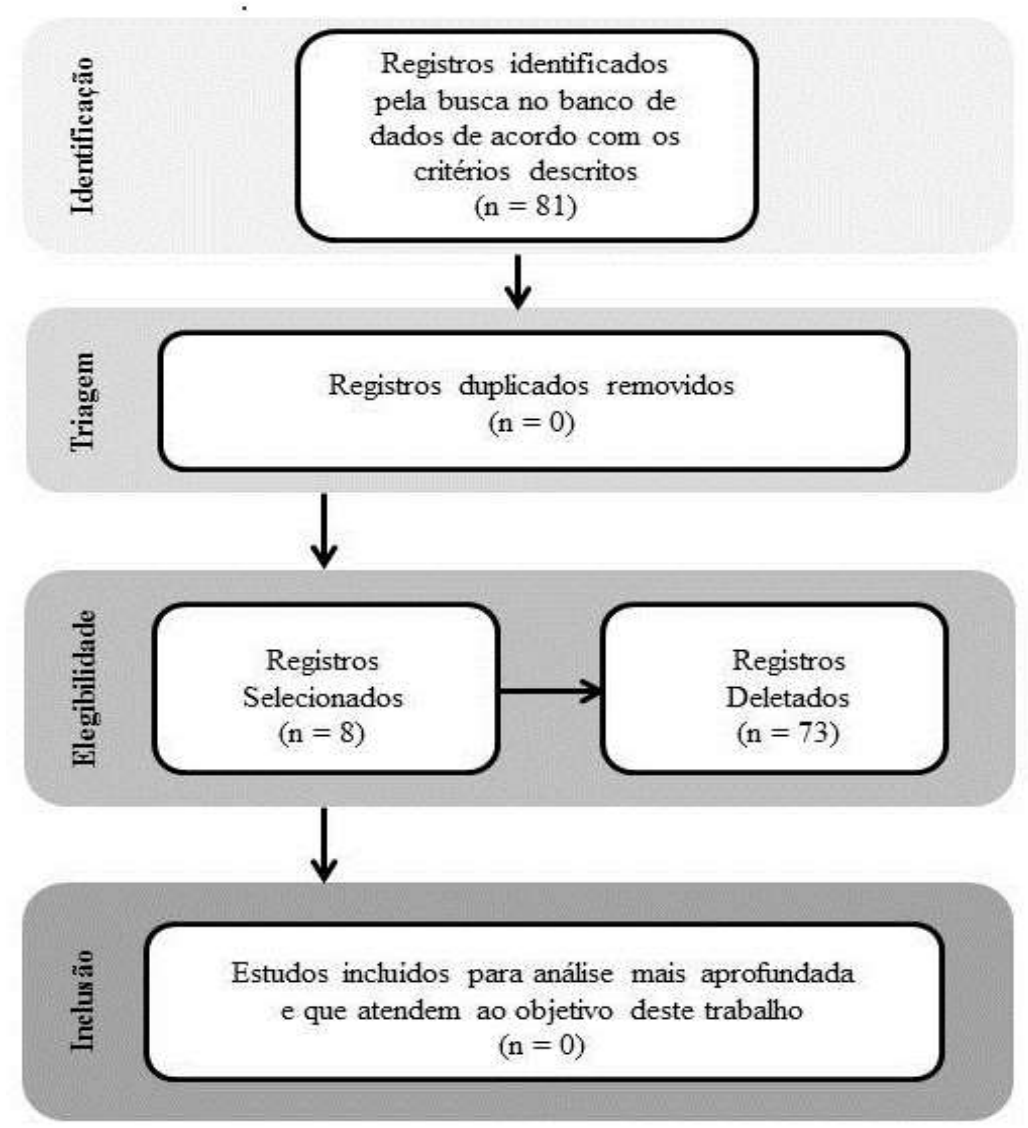

Fonte: Autores.

Dos artigos identificados nas bases de dados, após a leitura do título e do resumo foram excluídos os duplicados, os que não tinham nenhuma relação com plantas (mas que foram selecionados pelo uso do termo "wild", aparecendo resultados relacionados também a animais) e os que não tratavam de plantas alimentícias com usos para seres humanos. 
Os estudos selecionados para análise mais aprofundada foram lidos parcialmente e não foram identificados artigos científicos que tratassem de forma específica sobre espécies aquáticas silvestres com usos na alimentação, mas que também abordassem a composição nutricional de forma a assegurar a utilização segura da planta ou até mesmo amplamente utilizada para enriquecer as dietas das populações humanas. Aqueles artigos que citaram espécies terrestres e também aquáticas também foram excluídos, assim como, os que porventura tratam de espécies aquáticas, mas que não realizaram testes para avaliar a composição nutricional.

A Tabela 2 elenca espécies aquáticas utilizadas na alimentação em diversas regiões brasileiras de norte a sul do país. Dentre elas estão plantas silvestres, nativas, mas também aquelas que são exóticas, conhecidas popularmente como castanha d'água (Eleocharis dulcis) e flor de lótus (Nelumbo nucifera). Estas últimas podem afetar de o equilíbrio ecológico, pois podem tornar-se invasoras e nocivas às espécies nativas. No entanto, uma alternativa para auxiliar no controle da sua propagação seria utilizá-la na alimentação. 
Tabela 2: Plantas alimentícias não convencionais (PANC) aquáticas encontradas no Brasil.

\begin{tabular}{|c|c|c|c|c|}
\hline Nome popular & Família & Espécie & $\begin{array}{c}\text { Partes (s) utilizadas na } \\
\text { alimentação }\end{array}$ & Modo (s) de preparo \\
\hline Chapéu de couro & Alismataceae & Echinodurus macrophyllus (Kunth) Micheli & Folhas frescas e secas & Chá; refrigerante \\
\hline Couve d'água & Alismataceae & Limnocharis flava (L.) Buchenau & $\begin{array}{c}\text { Folhas com pecíolo e } \\
\text { inflorescências com } \\
\text { pedúndulo }\end{array}$ & $\begin{array}{l}\text { Flores empanadas; folhas refogadas e } \\
\text { cozidas }\end{array}$ \\
\hline Bredo d'água & Amaranthaceae & $\begin{array}{c}\text { Alternanthera } \\
\text { philoxeroides (Mart.) Griseb. }\end{array}$ & $\begin{array}{l}\text { Ramos foliares, ramos } \\
\text { jovens e folhas }\end{array}$ & Refogado; Gratinado \\
\hline Caeté do brejo & Cannaceae & Canna glauca $\mathrm{L}$. & Rizoma & Pasta, bolo, pudim e mingau do rizoma \\
\hline Castanha d'água & Cyperaceae & Eleocharis dulcis (Burm. f.) Trin. Ex Hensch. & Cormos & Leite, colmo torrado e em conserva \\
\hline Pinheirinho d'água & Haloragaceae & Myriophyllum aquaticum (Vell.) Verdc. & Ramos foliares & Refogado e preparação de bolinhos \\
\hline Buxixu; & Melastomataceae & Aciotis purpuracens (Aubl.) Triana & Folhas & Salada, geleia e refogado \\
\hline Flor de lótus & Nelumbonaceae & Nelumbo nucifera Gaertn. & Raízes tuberosas, núcula & $\begin{array}{l}\text { Purê e refogado das raízes; sementes } \\
\text { cozidas }\end{array}$ \\
\hline Vitória régia & Nymphaeceae & Victoria amazônica (Poepp.) J.E. Sowerby & Flor, sementes e rizoma & $\begin{array}{c}\text { Rizoma cozido; Canapés com pétalas; } \\
\text { pipoca das sementes }\end{array}$ \\
\hline Gigoguinha & Pontederiaceae & Heteranthera reniformis Ruiz \& Pav. & Ramos foliares e folhas & Refogada; bolinhos \\
\hline Manjubinha & Sphenocleaceae & Sphenoclea zeylanica Gaertn. & Ramos foliares e folhas & Bolinhos; risoto \\
\hline Taboa & Typhaceae & Typha domingensis Pers. & Palmito, rizoma e pólen & Palmito refogado; sopa dos rizomas \\
\hline Lírio do Brejo & Zingiberaceae & Hedychium coronarium J. Koenig & Rizomas, flores e botões & Saladas e rizoma frito \\
\hline
\end{tabular}

Fonte: Kinupp e Lorenzi (2014) 
As informações da tabela 2 foram extraídas do livro Plantas alimentícias não convencionais (PANC) no Brasil (Kinupp \& Lorenzi, 2014) e totalizam 13 espécies distribuídas em 12 famílias botânicas. Foram indicados os modos de preparo, valor nutricional (algumas espécies possuem estudos preliminares e identificação de componentes de interesse nutricional) e partes das plantas que podem ser inseridas na alimentação.

\section{Discussão}

\subsection{A lacuna na pesquisa sobre plantas alimentícias aquáticas e composição nutricional}

A pesquisa realizada não identificou estudos que contivessem ambas as palavras-chave "aquatic" e "nutricional composition" aliadas à temática sobre plantas alimentícias silvestres. Apesar do emprego específico, foram obtidos apenas artigos que se tratavam de um ou de outro termo demonstrando a escassa produção desta área.

No entanto, destaca-se o estudo de Cruz-Garcia et al. (2016), pois reforça a importância alimentar do cultivo convencional do arroz, alimento básico e gerador de renda de muitas populações, indicando a possibilidade de utilização também de plantas consideradas invasoras encontradas nas plantações dessas culturas. As plantas alimentícias silvestres possuem uma sazonalidade e estão distribuídas em ambientes terrestres e aquáticos. Neste último, os autores apontam Ipomoea aquatica e Marsilea crenata, espécies que são bastante utilizadas para alimentação de agricultores pobres.

A variedade de espécies conhecidas e utilizadas pelas populações tradicionais do Nordeste da Tailândia (Cruz-Garcia, et al, 2016) apontam a necessidade de lançar um olhar diferenciado para diversificação do tão bem estabelcido cultivo de espécies monoespecíficas que reduzem as possibilidades do conhecimento de alimentos biodiversos responsáveis, muitas vezes, por diminuir a vulnerabilidade provocada pela escassez de alimentos. Nascimento et al. (2012) tratam dos alimentos emergenciais consumidos no nordeste brasileiro e reforçam a ideia de que as plantas alimentícias silvestres são opções interessantes para proporcionar e garantir a segurança alimentar em qualquer parte do mundo.

As plantas alimentícias silvestres e/ou não convencionais apresentam-se como mais uma opção a problemática de falta de alimentos. No levantamento desenvolvido neste estudo, também é possivel destacar a pesquisa por Ogle et al. (2001), no qual são realizadas avaliações nutricionais de mulheres em comunidades situadas no Vietnã que utilizam amplamente espécies terrestres e aquáticas em seu hábito alimentar. Foram citadas 5 espécies e destaca-se que neste estudo também foi citada a ingestão de Ipomoea aquatica indicando alternativas para estudos relevantes e mais aprofundados sobre os seus componentes a fim de reafirmar o potencial alimentício já verificado por estas populações.

Dentre os estudos selecionados para análise do título e resumo verificou-se grande quantidade de estudos que abordam somente as plantas alimentícias silvestres de ambientes terrestres como as pesquisas desenvolvidas por Ceccanti et al. (2015), Aryal et al. (2018), Bathia et al. (2018), dentre outros, como os estudos brasileiros de Nascimento et al. (2013), Bortolotto et al. (2015) e Jacob et al. (2020).

O artigo escrito por Nascimento et al. (2013) trata da caracterização química de plantas alimentícias encontradas nas florestas do semi-árido nordestino, no entanto não são citadas espécies aquáticas tendo como possível justificativa os períodos de seca vivenciados por essa região do Brasil indicando a baixa possibilidade para que as populações utilizem espécies destes ambientes.

A verificação dos estudos excluídos proporcionou a vizualização de um cenário evidenciado por Jacob \& Albuquerque (2020) como lacunas no progresso das discussões sobre a temática de Alimentos biodiveros (termo utilizado pelos autores para se referir biodiversidade de plantas comestíveis, dentre elas estão as silvestres e as não convencionais, seja o uso total ou de partes de plantas com consumo convencional) a fim de evoluir para pesquisas que não somente identifiquem essas espécies, mas que possam também ser inseridas na alimentação. Para isso as mais recentes pesquisas desenvolvidas 
precisam adicionar dados de composição nutricional e os modos de preparona maioria das vezes relatados em estudos com enfoque etnobotânico.

O fluxograma representado na Figura 2, expõe as áreas de pesquisa que mais publicaram dentre os estudos científicos encontrados com os descritores "Food plants" and "wild" and "aquatic"e "Food plants" and "wild" and "nutritional composition" e propõe com base em Jacob e Albuquerque (2020), alternativa para o desenvolvimento de mais pesquisas sobre o potencial de espécies aquáticas.

Figura 2: Fluxograma com as áreas de pesquisa que mais publicaram e as possibilidades para o desenvolvimento de estudos científicos mais amplos sobre a temática.

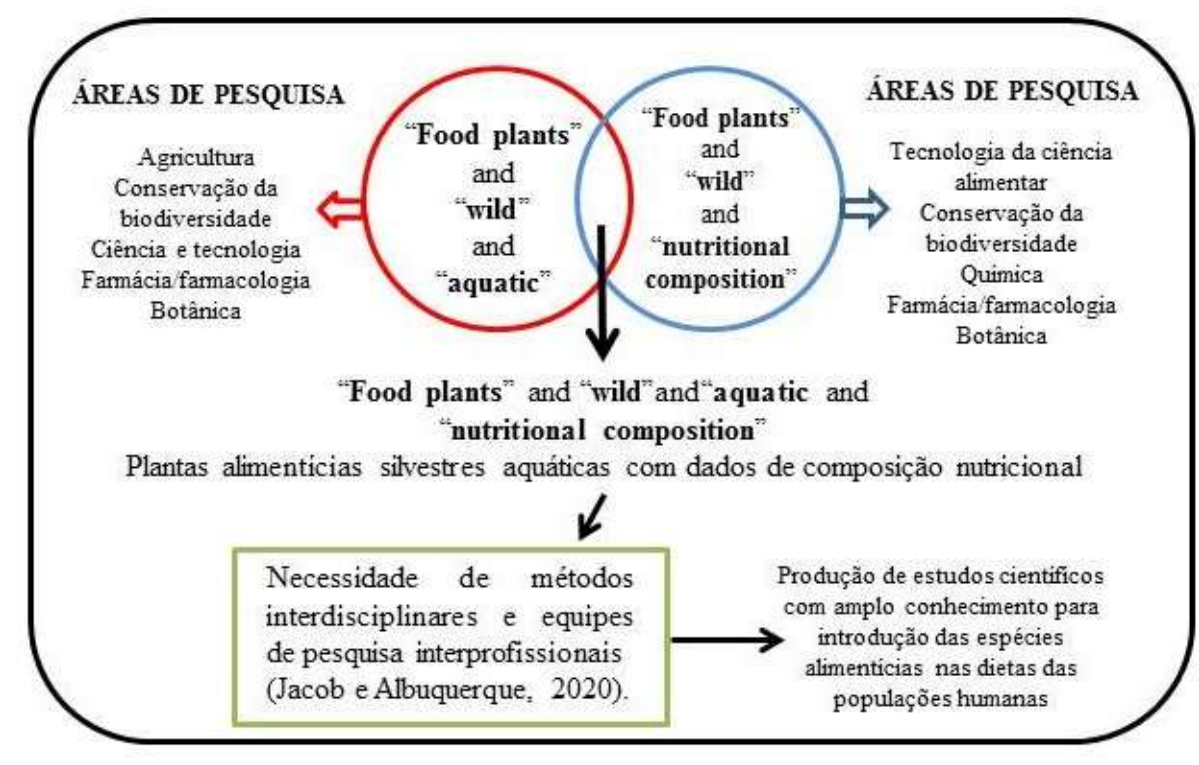

Fonte: Autores.

A perspectiva representada na Figura 2 demonstra que áreas de pesquisa sobre conservação da biodiversidade, Farmácia/farmacologia e botânica possuem interesse comum na investigação desta temática e ainda é possível ressaltar a química, a agricultura e tecnologia de alimentos. Cabe destacar a contribuição dos estudos etnobotânicos no acesso às informações sobre plantas alimentícias silvestres consumidas e validadas, em sua grande maioria, por poulações tradicionais partindo de relações homem-ambiente bastante relevantes para a ampliação do conhecimento científico.

\subsection{Indicações promissoras de plantas alimentícias aquáticas em publicações brasileiras}

As publicações brasileiras seguem os padrões identificados em estudos internacionais quando se refere a pesquisas realizadas abordando somente as espécies alimentícias sem a caracterização nutricional com ênfase para as plantas de ambiente de terrestre. No entanto, Kinupp e Barros (2008) apontaram três espécies aquáticas com rico potencial alimentício identificadas na região metropolitana de Porto Alegre, situada na região Sul do Brasil. A Althernanthera philoxeroides, Heteranthera reniformis e Typha dominguensis são também citadas em Kinupp e Lorenzi (2014) e foram incluídas na tabela 1.

A espécie Typha dominguensis é bem distribuída no território brasileiro e em diversos países, apresentando níveis elevados de proteína no palmito e no pólen com cultura agrícola promissora (Kinupp \& Barros, 2008). Já a Echinodorus macrophylus, considerada planta daninha é utilizada há bastante tempo em refrigerantes para conferir aroma. As preparações com a espécie Canna Glauca, planta nativa e bem distribuída no território brasileiro, vão desde a utilização dos rizomas em bolos ao pudim (Kinupp \& Lorenzi, 2014). 
As espécies exóticas e aquáticas também podem ser utilizadas na alimentação na fabricação de leite, refogados e saladas. No entanto, Eleocharis dulcis e Nelumbo nucifera precisam de mais estudos quanto aos seus constituinte e composição química para consumo seguro (Kinupp \& Lorenzi, 2014).

Apesar da grande disponibilidade e variedade de informações relevantes sobre a biodiversidade alimentar e principalmente as contribuições sobre plantas aquáticas relatadas por Kinupp e Lorenzi (2014), há ainda a lacuna quanto os dados nutricionais na maioria das espécies identificadas tanto terrestres como aquáticas. Entretanto, sob a perspectiva e objetivo deste estudo as informações relatadas por estes autores são as mais estruturadas e diversas referências encontradas para plantas aquáticas comestíveis.

\section{Conclusão}

A pesquisa relacionada a plantas alimentícias silvestres ainda é incipiente. No entanto, já existem movimentos de popularização do conhecimento e algumas espécies vegetais são utilizadas a nível local por comunidades tradicionais e difundidas para centros urbanos em feiras e mercados. Lançando um olhar às espécies aquáticas ou bem distribuídas neste ambiente, percebe-se com este estudo preliminar, a escassa produção científica nacional e internacional relacionada ao uso alimentício por populações humanas, a análise de constituintes químicos e composição nutricional para agregação destes alimentos em dietas ditas sustentáveis.

A metodologia utilizada para prospectar os artigos científicos foi relevante para a identificação de pesquisas com plantas aquáticas de uso alimentício, assim como aspectos nutricionais associados. Entretanto, os descritores e as bases de dados selecionadas podem não abranger toda a amplitude de pesquisas realizadas nesta área de estudo.

Os resultados aqui apresentados, mesmo que sejam indicativos preliminares, apontam para possibilidades de pesquisas promissoras sobre a composição nutricional de plantas alimentícias aquáticas com a finalidade de inseri-las futuramente no cardápio das populações, isoladas ou em associação com vegetais tradicionalmente inseridos na alimentação.

Tendo em vista o desconhecimento do potencial das plantas silvestres por parte dos pesquisadores, ressalta-se a grande contribuição que estudos etnobotânicos podem apresentar junto às comunidades, disponibilizando informações sobre o uso, modos de preparo e validação por parte dos habitantes que podem ter espécies vegetais aquáticas incorporadas ao seu hábito alimentar.

Vale ainda ressaltar que a ampliação dos estudos sobre os usos alimentícios de plantas silvestres aquáticas em pesquisas futuras pode estimular a sua valorização, conservação e incremento das opções alimentares, proporcionando segurança alimentar e nutricional, principalmente para as populações em situações de vulnerabilidade e com dietas pobres em micro e macronutrientes importantes na manutenção da qualidade de vida.

\section{Referências}

Aryal, K. P., Poudel, S., Chaudhary, R. P., Chettri, N., Chaudhary, P., Ning, W. \& Kotru, R. (2018). Diversity and use of wild and non cultivated edible plants in the Western Himalaya. Journal of Ethnobiology and Ethnomedicine, 14 (10): 1-18. doi 10.1186/s13002-018-0211-1

Bathia, H., Sharma, Y. P., Manhas, R. K. \& Kumar, K. (2018). Traditionally used wild edible plants of district Udhampur, J\&K, India. .Journal of Ethnobiology and Ethnomedicine, 14 (73): 1-13. 10.1186/s13002-018-0272-1

Bortolotto, I. M., Amorozo, M. C. M., Guarim Neto, G., Oldeland, J. \& Damasceno-Júnior, G. A. (2015). Knowledge and use of wild edible plants inrural communities along Paraguay River, Pantanal, Brazil. Journal of Ethnobiology and Ethnomedicine, 11 (46): 1-14. 10.1186/s13002-015-0026-2

Ceccanti, C., Landi, M., Incrocci, L., Pardossi, A., Venturi, F., Taglieri, I., Ferroni, G. \& Guidi, L. (2020). Comparison of Three Domestications and WildHarvested Plants for Nutraceutical Properties and Sensory Profiles in Five Wild Edible Herbs: Is Domestication Possible? Foods, 9: 1-20. $10.3390 /$ foods 9081065

Chaves, M. F. C., Morais, R. F. \& Barros, R. F. M. (2017). Práticas alimentares populares com uso de plantas silvestres: potencial para minimizar a insegurança nutricional no semiárido do Nordeste do Brasil. Gaia Scientia, 11 (2): 287-313. 
Research, Society and Development, v. 10, n. 3, e32510313457, 2021

(CC BY 4.0) | ISSN 2525-3409 | DOI: http://dx.doi.org/10.33448/rsd-v10i3.13457

Chaves, M. F. C. \& Barros, R.F.M. (2015). Cactáceas: recurso alimentar emergencial no semiárido, Nordeste do Brasil. Gaia scientia, 9 (2): $129-135$.

Cruz-García, G. S., Struik, P. C. \& Johnson, D. E. (2016). Wild harvest: distribution and diversity of wild food plants in rice ecosystems of Northeast Thailand. NJAS - Wageningen Journal of Life Sciences, 78: 1-11. 10.1016/j.njas.2015.12.003

Cruz, M. P., Medeiros, P., Sarmiento-Combariza, I., Peroni, N. \& Albuquerque, U. P. (2014). "I eat the manofê so it is not forgotten": local perceptions and consumption of native wild edible plants from seasonal dry forests in Brazil. Journal of Ethnobiology and Ethnomedicine, 10 (45): 1- 11. 10.1186/1746-4269$10-45$

Gomes, D.L., Ferreira, R. P. S., Santos, E. M. C., Silva, R. R. V. \& Medeiros, P. M. (2020). Local criteria for the selection of wild food plants for consumption and sale in Alagoas, Brazil. Ethnobiology and Conservation, 9 (10): 1-15. 10.15451/ec2020-04-9.10-1-15

Hora, J. S. L., Da Silva, T. C. \& Nascimento, V. T. (2020). "É natural, é bom! São frutos que vem da natureza": representações locais sobre o consumo de plantas alimentícias silvestres em uma área rural do Brasil. Ethnoscientia, 15. 10.22276/ethnoscientia.v5i1.286

Jacob, M. C. M. \& Albuquerque, U. P. (2020). Biodiverse food plants: Which gaps do we need to address to promote sustainable diets?.Ethnobiology and Conservation, 9 (9): 1-6. 10.15451/ec2020-04-9.09-1-6

Jacob, M. C. M., Medeiros, M. F. A. \& Albuquerque, U. P. (2020). Biodiverse food plants in the semiarid region of Brazil have unknown potential: A systematic review. Plos one, 15 (5): 1-24. 10.1101/2020.03.13.990358

Kinupp, V. F. \& Lorenzi, H. (2014). Plantas Alimentícias Não Convencionais (PANC) no Brasil: guia de identificação, aspectos nutricionais e receitas ilustradas. Instituto Plantarum de Estudos da Flora, São Paulo. 768 p.

Kinupp, V. F. \& Barros, I. B. I. (2008). Protein and mineral contents of native species, potential vegetables, and fruit. Ciência e Tecnologia de Alimentos, 28 (4): 846-857.

Nascimento, V. T., Lucena, R. F. P., Maciel, M. I. S. \& Albuquerque, U. P. (2013). Knowledge and Use of Wild Food Plants in Areas of Dry Seasonal Forests in Brazil. Ecology of Food and Nutrition, 52: 317 -343. 10.1080 / 03670244.2012 .707434

Nascimento, V. T., Vasconcelos, M. A. S., Maciel, M. I. S. \& Albuquerque, U. P. (2012). Famine Foods of Brazil's Seasonal Dry Forests: Ethnobotanical and Nutritional Aspects. Economic Botany, 66 (1): 23-34. 10.1007/s12231-012-9187-2

Ogle, B. M., Dung, N. N. X., Do, T. T. \& Hambraeus, L. (2001). The contribution of wild vegetables to micronutrient intakes among women: An example from the Mekong delta, Vietnam. Ecology of Food and Nutrition, 40 (2): 159-184. 10.1080/03670244.2001.9991646

Pereira A.S. et al. (2018). Metodologia da pesquisa científica. UFSM.

Raven, P. H., Evert, R. F. \& Eichhorn, S. E. (2014). Biologia Vegetal. (8a ed.), Editora Guanabara Koogan. 876 p. 